\title{
Detect Short Run of TWA Event with Time-Domain Algorithm
}

\author{
B Hostetler ${ }^{1}$, J Xue $^{2}$, B Young ${ }^{2}$, W Kaiser ${ }^{2}$, \\ M Findeis $^{2}$, D Gutterman ${ }^{1}$ \\ ${ }^{1}$ Medical College of Wisconsin, Milwaukee, WI, USA \\ ${ }^{2}$ GE Healthcare, Milwaukee, WI, USA
}

\begin{abstract}
T-wave alternans (TWA) has been associated with ventricular arrhythmias, and some of them are nonstationary, short-episode events (less than 128 beats). A specific time-domain algorithm for isolating $T$-wave alternans from ECG signals during exercise was developed. The purpose of this study was to test the hypothesis that the time-domain TWA algorithm can accurately quantify exercise TWA patterns, either long or short in duration, and evaluate the TWA measurement difference between time-domain and frequency-domain TWA methods.
\end{abstract}

\section{Introduction}

Microvolt $\mathrm{T}$ wave alternans (TWA) has been found useful to predict life-threatening arrhythmia events and therefore could also be useful in identifying candidates for implantable cardioverter defibrillators. Current $\mathrm{T}$ Wave Alternans (TWA) detection algorithms work in two different domains: time and frequency. Both methods try to detect microvolt TWA appearing on surface ECGs during stress testing. The time domain method has also been used on Holter data. Due to very small TWA amplitude changes and possible severe background noise including muscle noise, baseline wander, etc, it is very difficult to verify TWA detection from the displayed or the printed ECG waveforms. Special signal-to-noise ratio (SNR) improvements and signal processing are needed to enhance the SNR and to facilitate visualization of the TWA.

The frequency domain method uses spectral analysis to enhance the alternans pattern at the half of the beat frequency [1]. On the other hand, the time domain method enhances the SNR by using incremental median odd and even beats [2].

We have developed a simulation platform previously [3] to test the measurement accuracy of the algorithm objectively. It is important to have a direct and objective method to evaluate the microvolt TWA detection algorithms. With such a simulation platform, we should be able to evaluate TWA detection under different signal and noise circumstances.

In some clinical cases, the duration of alternate pattern of ST-T wave varied greatly, from as short as 20 beats to as long as hours. It is those short-run TWA events that can pose challenge to the detection algorithm. For example, the frequency-domain TWA method requires 128 beats for analysis, which assumes that the TWA event should be consistent for 128 beats..

A robust TWA detection algorithm should be able to pick short-run TWA as well as stable TWA. One of the design goals of the time-domain TWA method is to track short-run, or transient TWA events, while keeping a high specificity for noisy ECG signals.

\section{Methods}

DA comprehensive simulation program was built to generate different patterns of TWA with varying levels of signal and noise (based on MIT noise database). Noise was added to computer generated ECG signals with known TWA quantified by amplitude and phase. The test results were separated into two groups: low noise (noise < $200 \mathrm{uV}$ ) high noise (noise $>200 \mathrm{uV}$ ). The low and high noise groups are then compared to each other. The tests were also performed on some actual stress and Holter data with short episodes of TWA events.

\subsection{TWA detection algorithm}

(a) Time domain method - The basis for measuring TWA in the time domain is the formation of a median PQRST complex for odd and even beats [4]. The median beat complex is formed in a way to minimize the effects of random noise and allow comparison of the every-otherbeat $\mathrm{T}$ wave fluctuation by comparing ST measurement in the ST-T segment. The key process in this method is signal conditioning to reduce noise effects, rejection of ectopic beats, and formation of a median PQRST complex for the odd and even beat. The odd and the even median beats are updated by either a fixed increment, or a fraction 
of the difference between the median template and the incoming beat, whichever is less. The default update fraction is $1 / 32$, but other factors are selectable, namely $1 / 8,1 / 16$ or $1 / 64$. Figure 1 shows a diagram of this process. Figure 2 shows the results of this process for a simulated TWA series.

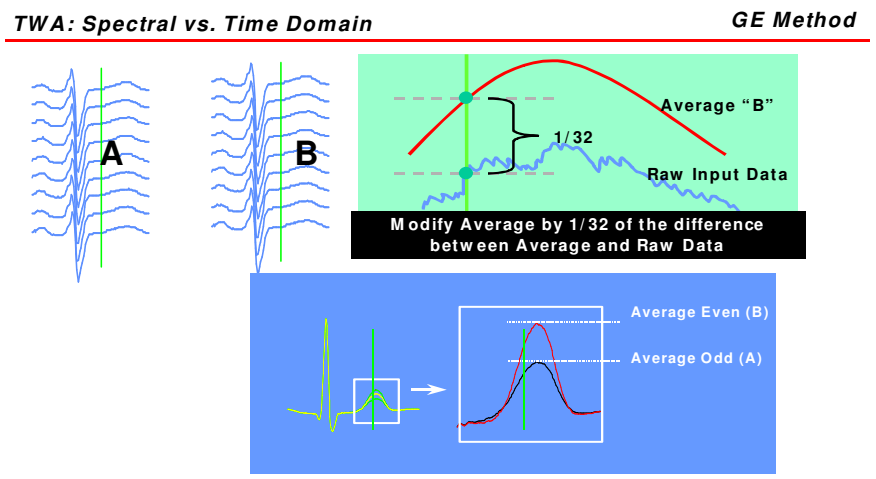

Figure 1. Incremental median used in the time do domain TWA method A/B.

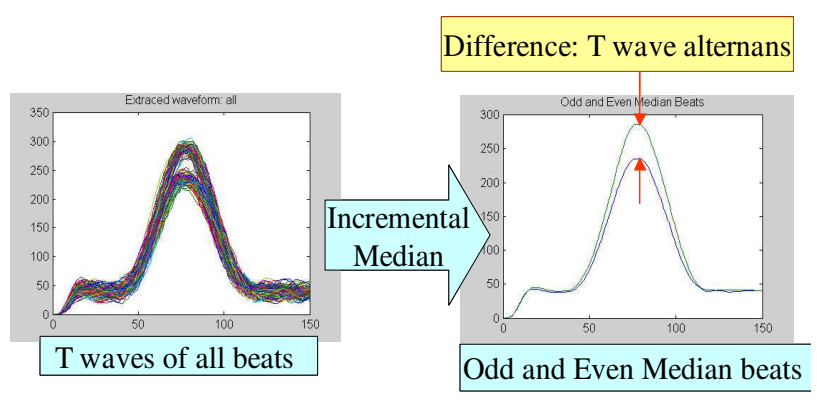

Figure 2. Time domain representation of TWA: Median even and odd beats.

(b) Frequency domain method: the details of this algorithm can be read from this reference [1]

\subsection{Simulation signal and model}

A simulation platform is designed for evaluation of TWA detection under different signal and noise circumstances. Some of the simulation parameters are:

- T wave model: The $\mathrm{T}$ wave is generated as: $A \cos ^{\alpha}\left(\frac{2 \pi t}{T}\right)$, where A controls the $\mathrm{T}$ wave amplitude, $\mathrm{T}$ controls the width, and power factor $\alpha$ controls the general shape of the $\mathrm{T}$ wave.
- TWA: TWA is added to alternate beats by changing the amplitude coefficient $\mathrm{A}$, or the power factor $\alpha$.

- Noise: Muscle noise and baseline wander were imported from MIT noise database[4]. The amplitude was changed based on signal-to-noise setting.

Figure 3 shows 2 simulated TWA pattern ECGs, (a) is low noise one, and (b) is high noise one.

Noise was added to computer generated ECG signals with known TWA quantified by amplitude and phase. The TWA amplitude varied from 0 to 33 microvolts measured as a maximum difference. The added noise contained two basic types: high frequency muscle noise and baseline wander varied from 50 to 1000 microvolts obtained from MIT noise database. This signal was then output to a standard 12 lead ECG by a custom designed ECG player. The CASE system (GEHC), which is a standard stress test machine, was used to analyze this data.

To study the effects of noise on the measurement of TWA, the test results are separated into two groups: relative clean (noise $<200 \mathrm{uV}$ ) noisy (noise $>200 \mathrm{uV}$ ). These detected TWA measurements with the same TWA inputs from the two groups are then compared to each other.

\subsection{Measurement comparison of Time-domain and frequency-domain methods}

In order to quantitatively evaluate the TWA measurement from the time-domain based method to the frequency-based method, the tests were repeated on the Cambridge Heart Stress system (CH-2000). The TWA measurements from both systems were compared. Muscle and baseline wander Noise from MIT database are added to the simulated TWA signals. The range of the noise is $50-1000 \mathrm{uV}$ in rms (root-mean-square). The analysis is performed on all cases, low-noise $(<200 \mathrm{uV})$, and high noise $(>=200 \mathrm{uV})$ cases for both time and frequency domain methods respectively. A regression routine is used to quantify relationship between the input TWA and the detected TWA from the two systems. The comparison between time and frequency domain systems was also performed. A regression routine is used to quantify the relationship between the TWA measurements of the two systems as well as identify the ratio of measured TWA values between the two systems.

\subsection{Actual Sampled stress ECGs}

Also included in the test are 22 data sets from ECG 
stress test, and 17 data sets from ESC_ST database of Physiobank [5]. Some short-run TWA event can be visualized from some of those data sets.

\section{Results}

\subsection{Time domain method: detected vs. simulated TWA amplitude}

The test results show the detected vs. simulated TWA for low noise, high noise, and combined cases, which respectively have the correlation coefficients of $0.98,0.99$ and 0.98 ; these also show a $0.92,0.82$, and 0.87 ratio of measured vs. simulated Peak-to-peak TWA amplitudes. Figure 4 shows measured vs. simulated TWA for combined noise level cases.

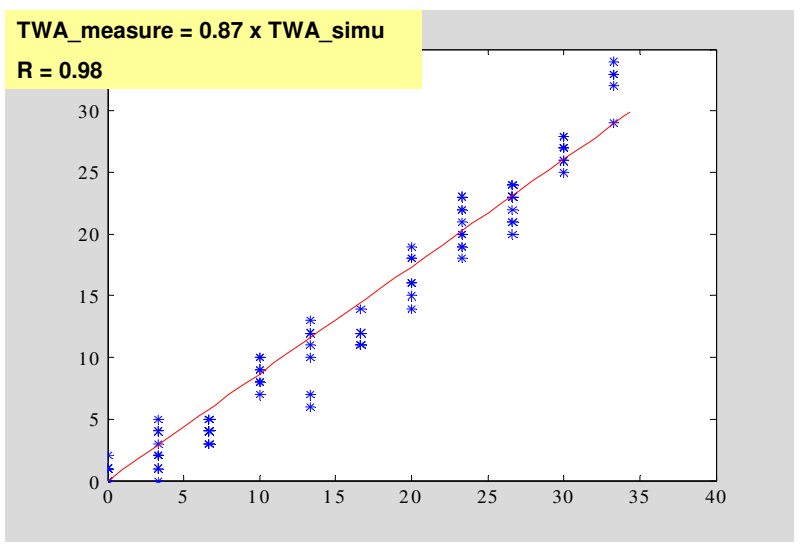

Figure 4. Time domain method: Measured vs. Simulated TWA amplitude.

\subsection{Frequency domain method: detected vs. simulated TWA amplitude}

The test results also show frequency-domain method detected vs. simulated TWA for low noise, high noise, and combined cases, which have the correlation coefficients of $0.99,0.92$ and 0.96 respectively; these linear regression show a 0.26 ratio of measured vs. simulated Peak-to-peak TWA amplitudes. Figure 5 shows frequency-domain method measured vs. simulated TWA for combined noise level cases.

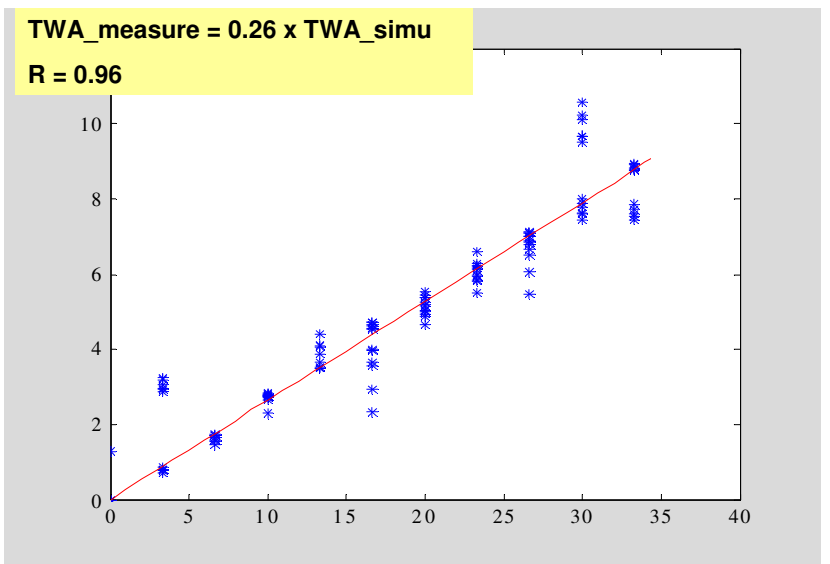

Figure 5. Frequency domain method: Measured vs. Simulated TWA amplitude.

\subsection{Time-domain vs. Frequency-domain method}

As we have noticed, there is a big difference in the regression ratio of measured vs. simulated TWA from the time-domain and frequency-domain methods. Therefore, it is useful to compare both measured TWA amplitudes directly using the two methods. Figure 6 is the comparison between the two methods with mixed noise levels. The measured TWA amplitude between time domain and frequency domain is $\mathbf{3 . 2 3}$.

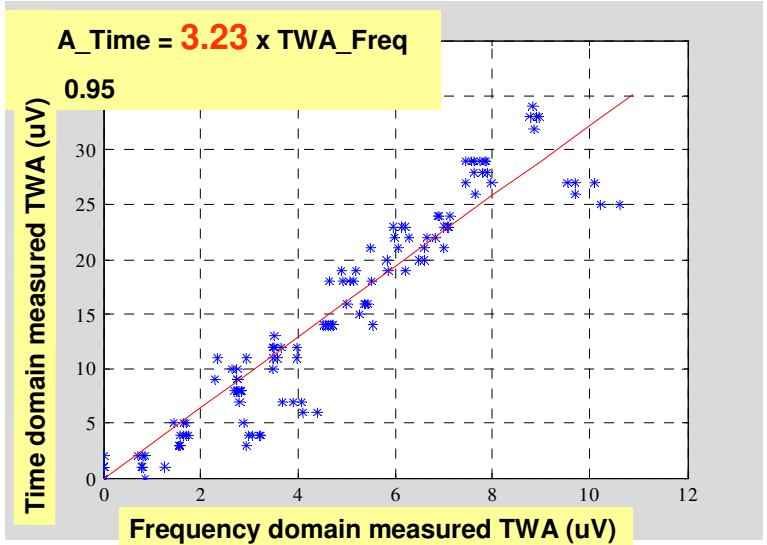

Figure 6. Measured TWA amplitude of Frequency vs. Time domain methods.

\subsection{Performance on short-run TWA event: Time vs. Frequency domain methods}

In the actual stress ECG data sets, there are cases that TWA patterns only last for a short period of time, for example, 20 beats. Figure 7 and 8 show two such cases, where TWA patterns can be seen directly from original 
ECG waveform, but it only last for about 20-30 beats, then back to non-alternans pattern. In both cases, the time-domain method clearly indicated TWA for A and B median patterns, while the frequency-domain method marked them as negative.

TWA Detection Example: Stress ECG
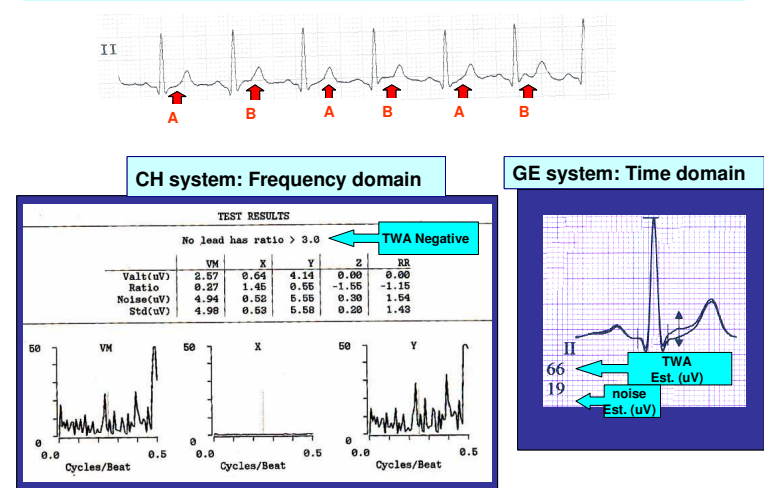

Figure 7. A short-run TWA event during stress test. Time domain method indicate a $66 \mathrm{uV}$ TWA amplitude in ST segment, while Frequency method mark as negative.

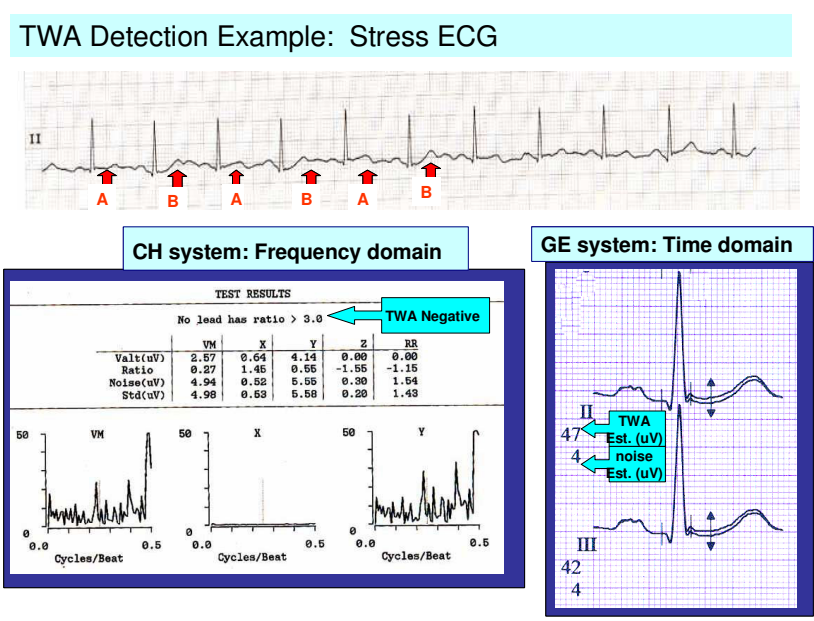

Figure 8. A short-run TWA event during stress test. Time domain method indicate a $47 \mathrm{uV}$ TWA amplitude in ST segment, while Frequency method mark as negative.

\section{Discussion and conclusions}

The test results show that the TWA signal can be reliably detected in both high-noise and low-noise groups by time-domain method, although the amplitude detected is $11 \%$ lower in high-noise group. The measured TWA of time-domain method is 3.23 larger than measured TWA of frequency domain method. The results also show that time-domain TWA method can detect short-run (that is less than 40 beats ) TWA activity better than the frequency-domain method.

Time-domain TWA method of GE CASE system is capable of identifying and distinguishing TWA from noise. The measured TWA amplitude from the timedomain TWA method decreases slightly as the noise increases. The measured TWA amplitude from frequency method is about 3 times smaller than that of time-domain method. The time-domain method therefore could detected short episodes of TWA better.

This study is the first to quantify the difference of TWA measurements from 2 major commercial systems: GE with time-domain method and Cambridge Heart $(\mathrm{CH})$ with frequency-domain method. The results showed that there is a large factor difference between the two systems. TWA measurements reported from time-domain method are about approximately 3 times as large as those of frequency domain method. The reason for this difference could be mainly due to average TWA across the entire T wave by the frequency domain method vs. the maximum TWA amplitude by time domain method. These results may be useful for guiding users of the two systems to set proper thresholds for positive TWA identification.

We also verified that the time-domain method is more sensitive to short -run TWA events ( fewer than 40 beats) than the frequency-domain method which needs 128 beats for processing.

\section{References}

[1] Rosenbaum DS, Jackson LE, . Smith JM, Garan H , Ruskin J, and Cohen RJ . Electrical alternans and vulnerability to ventricular arrhythmias. The New England J. Medicine, 330(4):235-241, January 271994.

[2] Nearing BD and Verrier RL. Modified moving average analysis of T-wave alternans to predict ventricular fibrillation with high accuracy. J. Appl Physiol. Vol 92, 541-549, 2002

[3] Xue JQ, Rowlandson GI, Effect of Noise on the Frequency and Time Domain Methods of T Wave Alternans. Proc. Computer in Cardiology 2005

[4] Kaiser W. et. al. Improving T-Wave Alternans Measurement Quality by Reducing Noise and Artifacts Computers in Cardiology 2004

[5] Goldberger AL, Amaral LAN, Glass L, Hausdorff JM, Ivanov PCh, Mark RG, Mietus JE, Moody GB, Peng CK, Stanley HE. PhysioBank, PhysioToolkit, and PhysioNet: Components of a New Research Resource for Complex Physiologic Signals. Circulation 101(23):e215-e220 [Circulation Electronic Pages;

Address for correspondence

Joel Xue

GE Healthcare

Menomonee Falls, WI 53051, U.S.A.

joel.xue@med.ge.com 